cyclisation. Similarly the reduction with small DNA molecules was not due to slower renaturation kinetics of small fragments resulting from their decreased flexibility. It is argued that reduced ring frequency with molecules less than $1-2 \mu \mathrm{m}$ is evidence for tandem repeats being spaced by non-repetitious sequences. Although Bick, Huang and Thomas (J. molec. Biol., 77, 75; 1973) tried to find evidence for the presence of this predicted non-repetitious DNA by examining rings prepared for electron microscopy in high concentrations of formamide, which would reveal unpaired single stranded regions within the terminal region of overlap, they were unable to do so. They concluded that either the non-repetitious sequences do not exist, which is unlikely, or they are too long or too short to be observed.

Similar conclusions concerning the interspersion of repetitive and nonrepetitive sequences have been reached by other groups using different techniques. For instance, Davidson et al. (J. molec. Biol., 77, 1-23; 1973) used labelled Xenopus DNA sheared to various known lengths denatured and then renatured, in the presence of excess cold Xenopus DNA of 450 nucleotides length, to $C_{o} t 50$, at which point predominantly repetitive sequences have renatured. They found that as the length of labelled DNA increases so a greater amount is bound to hydroxyapatite. Hydroxyapatite binds double stranded but not single stranded DNA at low salt concentrations and therefore allows their separation. In the conditions of the experiment any nonrepetitive sequences, although remaining single stranded, will bind to hydroxyapatite if they are covalently linked to renatured repetitive sequences.

The curve relating the proportion of ${ }^{3} \mathrm{H}$-labelled DNA fragments containing a repetitive element to fragment length shows three things. A steep linear increase ( $4 \%$ per 100 nucleotides) up to an inflexion point at about 700 nucleotides length and $60 \%$ fragments bound, followed by a slower linear increase $(0.7 \%$ per 100 nucleotides) up to 4,000 nucleotides length and $80 \%$ fragments bound. The first part of the curve does not extrapolate through zero bound at zero length but through about $20 \%$ representing the amount of repetitive DNA in the genome. Davidson et al. interpret these data on the basis of a model in which $50 \%$ of the Xenopus genome consists of repetitive elements about 300 nucleotides long interspersed with non-repetitive sequences 700-900 nucleotides long. A further $25 \%$ consists of repetitive sequences interspersed with longer ( $>4,000$ nucleotides) nonrepetitive sequences. The remainder is composed of unique sequences $(20 \%)$ without detectable repetitive elements and of tandem, low complexity repeti- tive sequences $(5 \%)$ without detectable non-repetitive elements. Similar experiments with sea urchin DNA (Graham et al., Cell, 1, 127; 1974) show that an identical model may be constructed. Furthermore, in two earlier papers (Kram et al., J. molec. Biol., 64, 103; 1972; Wu et al., ibid., 64, 211; 1972) on the organisation of repetitive sequences in Drosophila DNA other groups reached similar conclusions.

Although it seems likely on present evidence that a general model in which repeated sequences alternate with unique sequences in eukaryotic DNA can be constructed, a good deal more work is required to reveal in detail which repeating patterns are universal and to provide some notion of their relevance to genome function.

\section{Pattern formation in insects}

from our Insect Physiology Correspondent THERE are two ways in which the differentiated pattern of the living body may be supposed to arise. Each group of cells forming a given element in the body pattern may be derived from one progenitor cell, so that each component of the pattern represents a clone of cells which continues to propagate a persisting state of determination. Or, the cells forming an element of the body pattern may not have a different ancestry, or 'lineage', from the surrounding cells: their characters may have been determined by their position in the body. It can, of course, be argued that the second alternative is merely a repetition later in development of the same process by which the initial clones of the former alternative came into existence.

In normal development it seems clear that both these modes are concerned in the final generation of pattern. Many years ago, Sturtevant utilised the tendency of certain strains of Drosophila to form sexual mosaics (as the result of a spontaneous elimination of an X chromosome from somatic cells during embryonic development) in order to demonstrate the stage of development at which this mutation occurs and to relate this with the mosaic pattern in the resulting adult. This was done by coupling the mosaic strain with bristle characters that could be observed in the cuticle surface, and using the results to study the derivation or 'cell lineage' of the surface of the adult fly. Sturtevant found that related cells remain adjacent in patches without intermingling; that the occasional loss of an $\mathrm{X}$ chromosome happens at the early cleavage divisions; and that the mosaic patches of the opposite sex represent clones of these mutant cells.

Recently there has been a renewal of interest in this approach to the problem of differentiation. Garcia-Bellido et al. (Nature new Biol., 245, 251; 1973) used somatic crossing-over induced by $\mathrm{X}$ rays to generate marked clones in Drosophila in which he could follow the distribution of characters in the cuticle of the wing surface. The resultant wings were made up of mixed clones, some of marked cells homozygous for the wild type allele of minute, $\mathrm{M}^{+} / \mathrm{M}^{+}$; others, the unmarked cells of the heterozygous strain, $M^{+} / M$. The marked cells have a much higher growth rate than the unmarked heterozygote. But in spite of this they did not overgrow the unmarked cells. It turned out that the wing is subdivided into eight compartments, and, provided i clone is generated after the time of final subdivision, it is strictly confined to one compartment. But within this compartment the cells could be differentiated by local control so as to contribute to the ordinary wing surface, to parts of wing veins, or to marginal bristles-depending not on cell lineage but on position at the time of differentiation.

Lawrence (J. Embryol. exp. Morph., 30, 681; 1973) now describes experiments in this same general field carried out on the milkweed bug Oncopeltus. $\mathrm{He}$ deals solely with the integument of the abdomen. Mild X irradiation of the egg led to somatic mutations which resulted in changes in the pterin pigments of the epidermal cells from the normal orange to deep orange, transparent, white or pink. As in Sturtevant's experiments these mutations resulted in clonal patterns of epidermal cells which are readily recognised by their unusual pigmentation. The results provide much information about the timing of determination for the structure of the abdomen, the number of cells which furnish the ancestry for a single abdominal segment and so forth. Again it is evident that the clones are subject to strict control. If the ancestral cell mutates after segmentation of the presumptive abdomen has been determined (during blastoderm formation), the clones are restricted to a single quadrant of a segment (dorsal or ventral, left or right). The daughter cells forming the members of a clone tend to hold together in a clump, but they can mix with their neighbours and the groups become fractionated to some extent. On the other hand, they cannot cross the boundaries between segments, between dorsal and ventral, or across the midline of the body.

The effective boundaries between segments are established at the time, 10-24 $\mathrm{h}$ after egg laying, when the limiting membranes of the cells are developing in the blastoderm. The nature of these and other invisible boundaries between compartments will be a key question in the understanding of differentiation. 